(A)

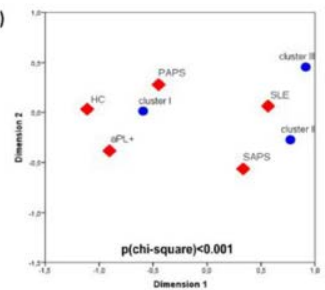

(B)
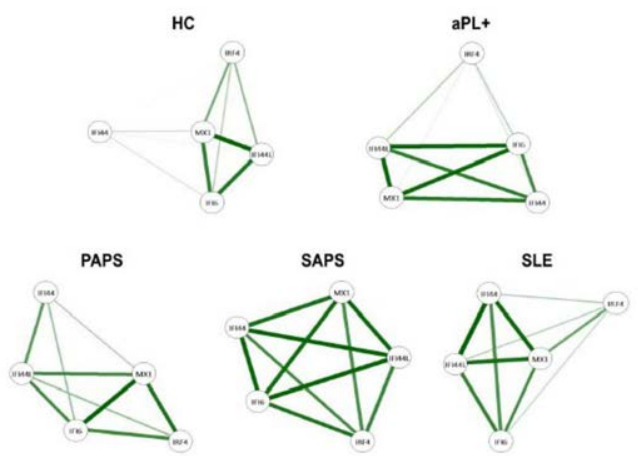

Figure 2. Panel A) Clusters analysis, correlated with clinical status of the patients by correspondence analysis ( $p<0.0001)$. Panel B) Network analyses of the interferon regulated genes networks among groups.

Acknowledgments: None

Disclosure of Interests: None declared

DOI: 10.1136/annrheumdis-2020-eular.4568

\section{OP0049 EFFICACY OF ANIFROLUMAB IN ACTIVE SYSTEMIC LUPUS ERYTHEMATOSUS: PATIENT SUBGROUP ANALYSIS OF BICLA RESPONSE IN 2 PHASE 3 TRIALS}

E. F. Morand ${ }^{1}$, R. Furie ${ }^{2}$, Y. Tanaka ${ }^{3}$, R. Kalyani ${ }^{4}$, G. Abreu ${ }^{5}$, L. Pineda ${ }^{4}$, R. Tummala ${ }^{4} .{ }^{1}$ Monash University, Melbourne, Australia; ${ }^{2}$ Zucker School of Medicine at Hofstra/Northwell, Great Neck, United States of America; ${ }^{3}$ University of Occupational and Environmental Health, Japan, Kitakyushu, Japan; ${ }^{4}$ BioPharmaceuticals Medical, AstraZeneca, Gaithersburg, United States of America; ${ }^{5}$ BioPharmaceuticals Medical, AstraZeneca, Gothenburg, Sweden

Background: Treatment of patients with systemic lupus erythematosus (SLE) with the type I interferon (IFN) receptor inhibitor anifrolumab resulted in higher British Isles Lupus Assessment Group (BILAG)-based Composite Lupus Assessment (BICLA) response rates vs placebo at Week 52 in the phase 3 randomized trials, TULIP-2 (primary endpoint; $16.3 \%$ difference) ${ }^{1}$ and TULIP-1 (secondary endpoint; $16.4 \%$ difference). ${ }^{2}$ BICLA is a validated composite global disease measure that registers both partial and complete improvement within organ systems.

Objectives: TULIP-2 and TULIP-1 data were analyzed to evaluate BICLA responses to anifrolumab vs placebo at Week 52 in protocol-defined subgroups of patients with active SLE.

Methods: TULIP-2 and TULIP-1 were randomized, double-blind, placebo-controlled trials that evaluated efficacy and safety of intravenous anifrolumab vs placebo administered every 4 weeks, with the primary endpoints assessed at Week 52, in patients with moderate to severe SLE despite standard-of-care treatment. ${ }^{1,2}$ BICLA responses are defined by all of the following: reduction of baseline BILAG-2004 A and B domain scores to B/C/D and C/D, respectively, and no worsening in any organ system; no worsening of the SLE Disease Activity Index 2000 (SLEDAI-2K) score; no worsening of $\geq 0.3$ points in the Physician's Global Assessment (range 0-3); no trial treatment discontinuation; and no use of medications restricted by the protocol. ${ }^{3}$ BICLA responses were compared between anifrolumab $300 \mathrm{mg}$ and placebo groups, and robustness of BICLA responses was assessed across protocol-defined subgroups. TULIP-1 data were analyzed incorporating the amended restricted medication rules, as described. $^{2}$

Results: In TULIP-2 and TULIP-1, 180 patients in each trial received anifrolumab $300 \mathrm{mg}$ (182 and 184 patients received placebo, respectively). Baseline demographics, disease characteristics, and standard-of-care medications were balanced between anifrolumab and placebo groups within both TULIP trials. Patients in TULIP-2 and TULIP-1 had comparable BICLA responses (Figure). Across multiple subgroups, higher percentages of patients achieved BICLA responses at Week 52 in the anifrolumab vs placebo arms (Figure). There was concordance of BICLA responses favoring anifrolumab across the protocol-defined subgroups of baseline disease severity (SLEDAI-2K <10 points [difference 15.3\%, TULIP-2; 16.9\%, TULIP1] vs $\geq 10$ points [difference $16.7 \%$, TULIP-2; $17.1 \%$, TULIP-1]) and baseline oral corticosteroid use (prednisone or equivalent $<10 \mathrm{mg} / \mathrm{d}$ [difference $20.1 \%$, TULIP-2; $16.2 \%$, TULIP-1] vs $\geq 10 \mathrm{mg} / \mathrm{d}$ [difference $12.0 \%$, TULIP-2; $17.7 \%$, TULIP-1]). Numerically different BICLA effect sizes between the anifrolumab vs placebo arms were observed in both studies in relation to baseline IFN gene signature status (high [difference 17.3\%, TULIP-2; 19.1\%, TULIP-1] vs Iow [difference $11.2 \%$, TULIP-2; 7.5\%, TULIP-1]). Other subgroups including age, sex, age at onset, race, and anti-drug antibody status showed similar uniformity of response.

Conclusion: The uniformity of robust BICLA response rates across prespecified subgroups in both phase 3 trials shows consistent clinical benefit of anifrolumab irrespective of patient baseline characteristics. However, given the small patient numbers in some subgroups, these results should be interpreted with caution.

\section{References:}

[1] Morand EF, et al. N Engl J Med. 2020;382:211-221.

[2] Furie RA, et al. Lancet Rheumatol. 2019;1:e208-e219.

[3] Wallace DJ, et al. Ann Rheum Dis. 2014;73:183-190.

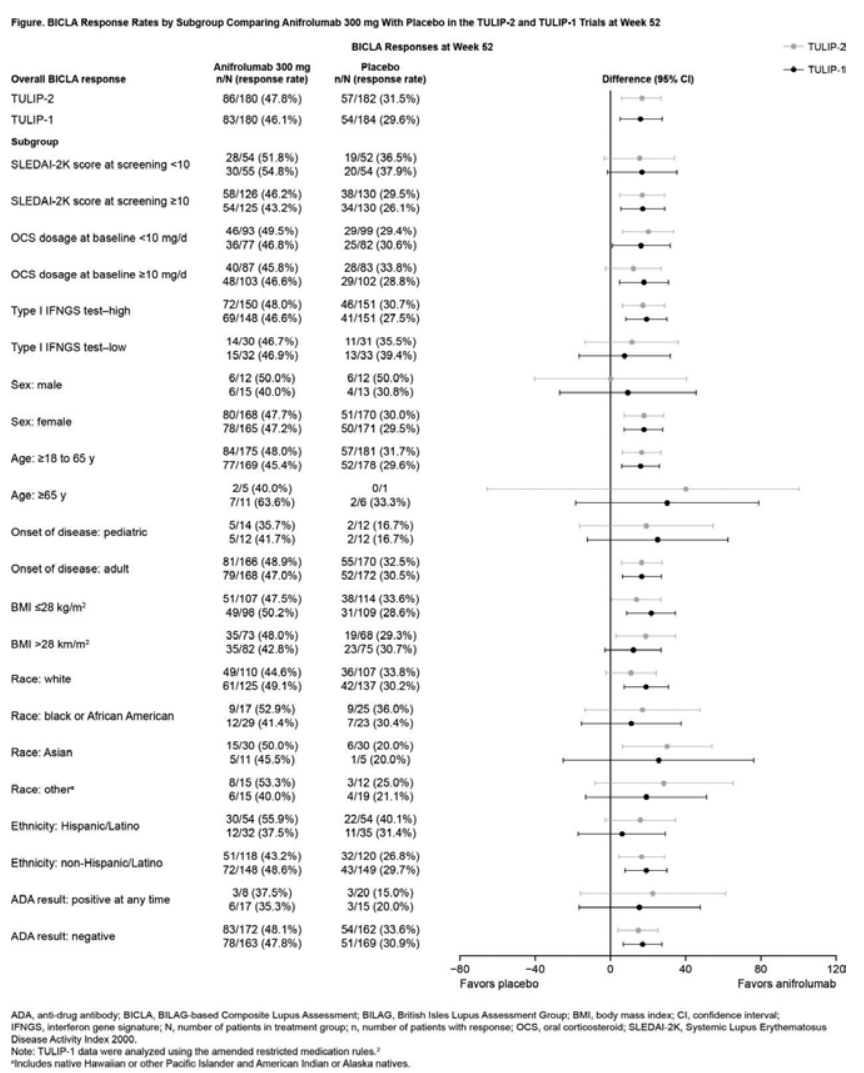

Disclosure of Interests: Eric F. Morand Grant/research support from: AstraZeneca, Consultant of: AstraZeneca, Speakers bureau: AstraZeneca, Richard Furie Grant/research support from: AstraZeneca, Biogen, Consultant of: AstraZeneca, Biogen, Yoshiya Tanaka Grant/research support from: Asahi-kasei, Astellas, Mitsubishi-Tanabe, Chugai, Takeda, Sanofi, Bristol-Myers, UCB, Daiichi-Sankyo, Eisai, Pfizer, and Ono, Consultant of: Abbvie, Astellas, Bristol-Myers Squibb, Eli Lilly, Pfizer, Speakers bureau: Daiichi-Sankyo, Astellas, Chugai, Eli Lilly, Pfizer, AbbVie, YL Biologics, Bristol-Myers, Takeda, Mitsubishi-Tanabe, Novartis, Eisai, Janssen, Sanofi, UCB, and Teijin, Rubana Kalyani Employee of: AstraZeneca, Gabriel Abreu Employee of: AstraZeneca, Lilia Pineda Employee of: AstraZeneca, Raj Tummala Employee of: AstraZeneca

DOI: 10.1136/annrheumdis-2020-eular.3557 